University of Nebraska - Lincoln

DigitalCommons@University of Nebraska - Lincoln

May 1981

\title{
Detection of vibrationally excited nitrogen by trapped electron and electron transmission methods
}

\author{
J.A. Michejda \\ Engineering and Applied Science Department, Yale University, New Haven, Connecticut \\ L.J. Dube \\ Engineering and Applied Science Department, Yale University, New Haven, Connecticut \\ Paul Burrow \\ University of Nebraska - Lincoln, pburrow1@unl.edu
}

Follow this and additional works at: https://digitalcommons.unl.edu/physicsburrow

Part of the Physics Commons

Michejda, J.A.; Dube, L.J.; and Burrow, Paul, "Detection of vibrationally excited nitrogen by trapped electron and electron transmission methods" (1981). Paul Burrow Publications. 23.

https://digitalcommons.unl.edu/physicsburrow/23

This Article is brought to you for free and open access by the Research Papers in Physics and Astronomy at DigitalCommons@University of Nebraska - Lincoln. It has been accepted for inclusion in Paul Burrow Publications by an authorized administrator of DigitalCommons@University of Nebraska - Lincoln. 


\title{
Detection of vibrationally excited nitrogen by trapped electron and electron transmission methods
}

\author{
J. A. Michejda and L. J. Dubé \\ Engineering and Applied Science Department, Yale University, New Haven, Connecticut 06520 \\ P. D. Burrow \\ Behlen Laboratory of Physics, University of Nebraska, Lincoln, Nebraska 68588
}

(Received 22 February 1980; accepted for publication 6 January 1981)

\begin{abstract}
Two techniques for detection of vibrationally excited nitrogen using low-energy electron scattering are described. The first technique uses the trapped electron method to observe excitation from $N_{2}^{v}$ into the $B{ }^{3} \Pi_{g}$ state near threshold. From the known Franck-Condon factors, the contribution from excited vibrational levels may be unfolded. The second technique employs the electron transmission method to detect sharp structure in the total scattering cross section resulting from the formation of temporary negative ions. Because of uncertainties in the total scattering cross sections from each of the vibrational levels, the transmission method is less accurate than the trapped electron technique. As a consistency check, the vibrational temperature of $\mathrm{N}_{2}$ downstream from a microwave discharge is determined as a function of the discharge power. Within their respective error limits, the two methods are in good agreement.
\end{abstract}

PACS numbers: $35.80 .+$ s, 34.80.Gs.

\section{INTRODUCTION}

A number of methods have been employed to detect vibrationally excited nitrogen molecules. The techniques differ greatly in their sensitivity and complexity, in the environments in which they can be applied, and in the ease with which measurements may be interpreted. A partial list of methods would include calorimetry, ' ultraviolet absorption, ${ }^{2}$ mass spectrometry, ${ }^{3}$ photoionization, ${ }^{4}$ infrared tracer techniques, ${ }^{5}$ emission spectra produced by Penning ionization $^{6}$ and electron impact, ${ }^{7}$ Raman scattering, ${ }^{8}$ coherent anti-stokes Raman spectroscopy, ${ }^{9}$ photoelectron spectroscopy, ${ }^{10}$ electron energy-loss spectra, ${ }^{11}$ and others.

In this paper we describe two techniques for detecting vibrationally excited $\mathrm{N}_{2}$ using low-energy electron scattering. Although the development of new diagnostic techniques is undoubtedly of importance in its own right, our motivation stems from an interest in electron collisions with excited molecules. Since we were equipped to carry out studies of several types of collision processes, it was desirable to see if any of the methods could be used in a purely diagnostic manner to determine the density and vibrational distribution of excited molecules produced by an external source. Our apparatus therefore would serve both to characterize the species under study and to investigate other collision processes with the excited molecules. This experimental arrangement has at least two advantages: first, it avoids having to incorporate additional complex equipment into our apparatus, and second, the density of excited molecules is determined precisely where we need to know it, namely, at the location of the electron beam.

This approach has proved feasible and we have carried

\footnotetext{
${ }^{a}$ Present address: Bell Laboratories, Murray Hill, Pennsylvania 07974 'Present address: Department of Physics, The University of Freiburg, Freiburg, Germany.
}

out a study of the ionization cross sections near threshold of vibrationally excited $\mathrm{N}_{2}$ which will be reported elsewhere. ${ }^{12}$ In the present paper we will discuss only the diagnostic aspects of this work.

Briefly, the detection techniques we have utilized are based on the trapped electron ${ }^{13}$ and electron transmission ${ }^{14}$ methods. In the first case, certain characteristics of the excitation by electron impact from the vibrational levels of the ground electronic state of $\mathrm{N}_{2}$ into the $B^{3} \Pi_{\mathrm{g}}$ state near threshold are exploited. The advantage of this method is that it requires only the well-known Franck-Condon factors between the two states to extract the relative vibrational populations. The second technique, electron transmission, is sensitive to sharp structure in the total electron scattering cross section resulting from the formation of temporary negative ions. When applied to vibrationally excited $\mathrm{N}_{2}$, additional sharp structure appears in the transmission spectrum. To unfold the vibrational distribution, the total resonant scattering cross section for each vibrational level is required over the energy range in which $\mathrm{N}_{2}^{-}\left(X^{2} \Pi_{g}\right)$ is formed. Using the cross sections computed by Dubé and Herzenberg, ${ }^{15}$ we find that the results obtained with this diagnostic method are in good agreement with those determined by the more straightforward trapped electron and technique.

In the following sections, we describe first the apparatus, and then the trapped electron and transmission methods. Finally, a general discussion of the relative merits of each technique is given.

\section{APPARATUS}

The apparatus described in this section was designed for the investigation of electron scattering processes from a variety of excited species as well as free radicals. An earlier version has been discussed elsewhere. ${ }^{16}$ Schematic drawings of the main vacuum chamber and the gas-flow system are shown in Figs. 1 and 2, respectively. 


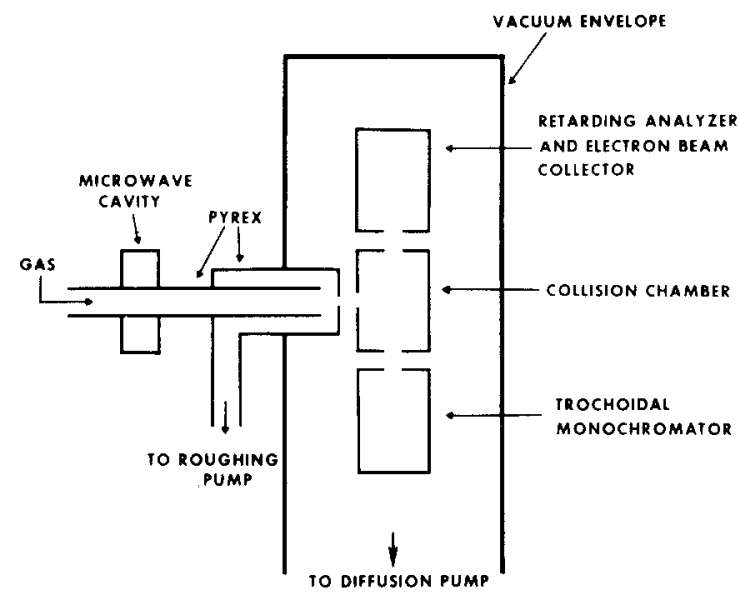

FIG 1. Schematic drawing of the primary components of the apparatus.

\section{A. Source for vibrationally excited $\mathbf{N}_{2}$}

A microwave discharge at $2450 \mathrm{MHz}$ in rapidly flowing $\mathrm{N}_{2}$ is used as a source of vibrationally excited $\mathrm{N}_{2}$ molecules, hereafter abbreviated as $\mathrm{N}_{2}^{\mathrm{v}}$. The major part of the gas flow takes place in 14-mm Pyrex tubing at a typical pressure of 6 Torr. The gas-flow velocity in this configuration was approximately $50 \mathrm{~m} / \mathrm{s}$. A small portion of the gas is sampled by effusion through a narrow slit, 0.06 by $10 \mathrm{~mm}$, in a thin Pyrex plate at the end of the reentrant portion of the tubing.

The $\mathrm{N}_{2}$ afterglow produced downstream from the discharge is a complex phenomenon and has been surveyed by Wright and Winkler. ${ }^{17}$ For the purpose of the present work, it is sufficient to summarize the production of excited species as follows. Although the active discharge produces both electronically excited molecules and atoms directly, none of these survive in measurable amounts downstream from the

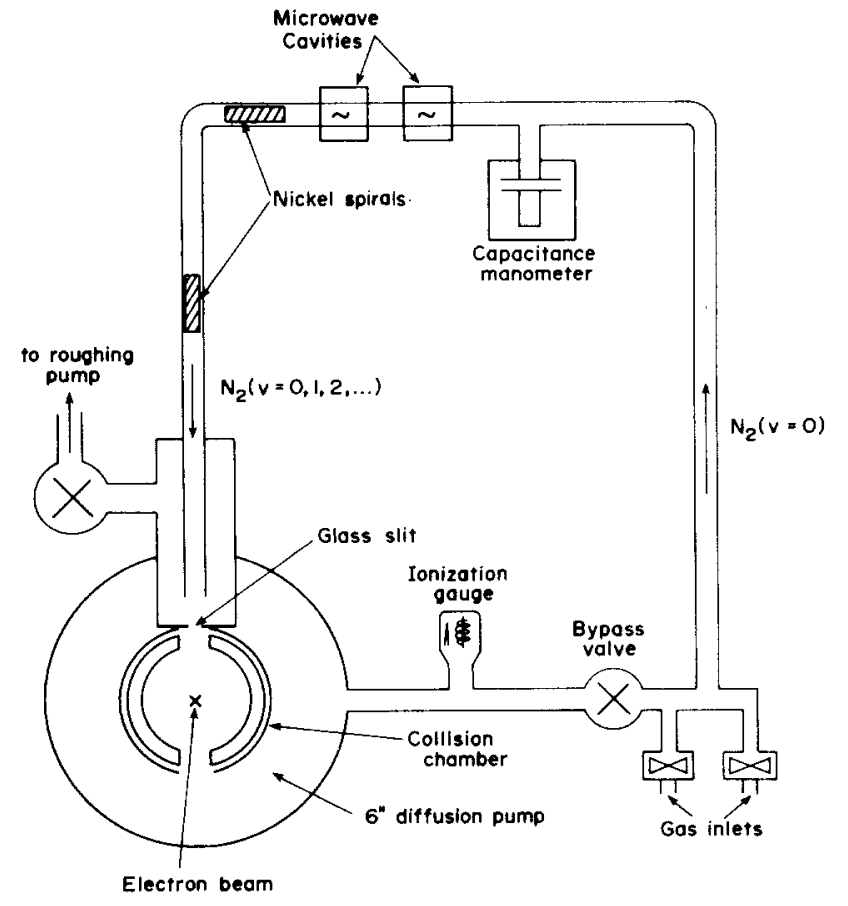

FIG 2. Schematic drawing of the gas flow system. discharge for times greater than a few milliseconds. ${ }^{3}$ Nitrogen atoms in the ground state, however, are produced in amounts typically a few percent of the total gas density, and by recombination serve to regenerate other electronically excited species which comprise the long-studied active nitrogen afterglow. The remaining constituent produced copiously by the discharge is $\mathrm{N}_{2}^{v}$.

To eliminate all excited species except $\mathbf{N}_{2}^{v}$, it was therefore necessary to remove the atoms from the afterglow. This was carried out by utilizing the property that atoms recombine on metal surfaces at a much faster rate than $\mathrm{N}_{2}^{v}$ molecules deactivate. ${ }^{17.18}$ Two nickel cylinders of length $2 \mathrm{~cm}$ were used inside the glass tubing downstream from the discharge. Each cylinder contained a strip of nickel foil rolled into a spiral to maximize the area of interaction with the gas while not reducing the conductance of the tubing substantially. The removal of the atomic species was signaled by the absence of the yellow afterglow characteristic of the gas phase atom recombination. In the present experiments, the discharge source was typically placed $75 \mathrm{~cm}$ from the electron collision chamber, producing transit times of about 15 msec.

\section{B. Main vacuum chamber}

The electron gun is housed inside a stainless-steel vacuum envelope and pumped by a 6 in. oil diffusion pump topped with a liquid-nitrogen trap. The background pressure obtained in the system with no gas entering is $2 \times 10^{-8}$ Torr.

With the discharge operating at 6 Torr, the background pressure in the scattering region is approximately $10^{-3} \mathrm{Torr}$. The density of the molecular beam in the center of the electron scattering region, however, is about 25 times greater than the background value.

\section{The electron beam apparatus}

An expanded view of the electron gun is illustrated in Fig. 3. All metal parts of the gun, except for the filament and support pieces, are machined from a nonmagnetic coppernickel alloy. To minimize contact potential variations along the surfaces, the electrodes are gold-plated.

The electron monochromator, plates M1-M7, is in the trochoidal geometry similar to the design described in detail by Stamatovic and Schulz. ${ }^{19}$ An axial magnetic field of typically $80-200 \mathrm{G}$, produced by external Helmholtz coils, is used to collimate the beam. An electron beam current of approximately $10^{-9}-10^{-8} \mathrm{~A}$ is achieved with energy resolution between 40 and $60 \mathrm{meV}$ full width at half maximum.

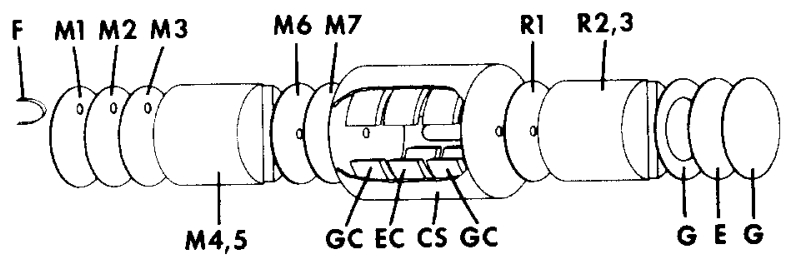

FIG. 3. Schematic diagram of the electron beam apparatus. 
The outer envelope of the collision chamber is formed by the electron beam entrance and exit electrodes joined by a thin cylindrical shell (CS). Two openings running parallel to the tube axis are machined out in the shell to allow passage of the molecular beam through the electron scattering region. To prevent the penetration of external potentials, the wide exit opening is covered with a $90 \%$ transparent molybdenum mesh.

The interior of the collision chamber is composed of three electrically insulated cylinders GC and EC, each of which is split into two C's to permit passage of the molecular beam. By applying the appropriate potentials to the split cylinders with reference to the outer envelope, the various modes of operation described below may be carried out.

The remaining portions of the gun $R$ 1-3 are used to retard the main electron beam to low energy for the transmission experiments described below, and to prevent electrons which are reflected from the main beam collector $(E)$ from re-entering the collision chamber.

In the narrow gap between the entrance to the collision chamber and the exit slit in the glass tubing, we found it useful to place two small electrodes such that a transverse electric field could be applied to the gas coming from the discharge. The deflecting field minimized considerably the stray current reaching the collision chamber.

\section{DETECTION OF N2 BY THE TRAPPED ELECTRON METHOD}

\section{A. The trapped electron method}

The trapped electron method, devised by Schulz, ${ }^{13}$ has been amply discussed in the literature ${ }^{20}$ and only a brief review will be provided here. The apparatus is designed to collect with high efficiency the slow electrons which are produced when the energy of the incident electron beam is just above the threshold for an inelastic collision process. By biasing the outer portion of the collision chamber slightly negative with respect to the inner chamber plate, an electrostatic potential well of depth $W$ (volts) is produced along the tube axis. If an electron whose incident kinetic energy inside the collision chamber is $e\left(V_{0}+W\right)$ makes an inelastic collision and suffers an energy loss greater than $\mathrm{eV}_{0}$, it cannot escape through the entrance or exit apertures and will be trapped inside. The trapped electrons spiral back and forth following the axial magnetic field lines and eventually make a sufficient number of elastic collisions with the background gas to diffuse across the field lines and be collected on the inner chamber plates. Electrons which have lost an energy less than $\mathrm{eV}_{0}$ will not be trapped, since their residual energy is sufficient to overcome the potential barrier at the ends of the collision chamber. Thus, as a function of the incident electron energy, the trapped electron current is zero just below an inelastic threshold, grows to a peak whose height is proportional to the magnitude of the inelastic cross section at $e W$ above threshold, and then drops to zero for incident energies greater than $e W$ above the inelastic threshold.

Two aspects of the collision chamber design may be of interest with regard to these measurements. In experiments

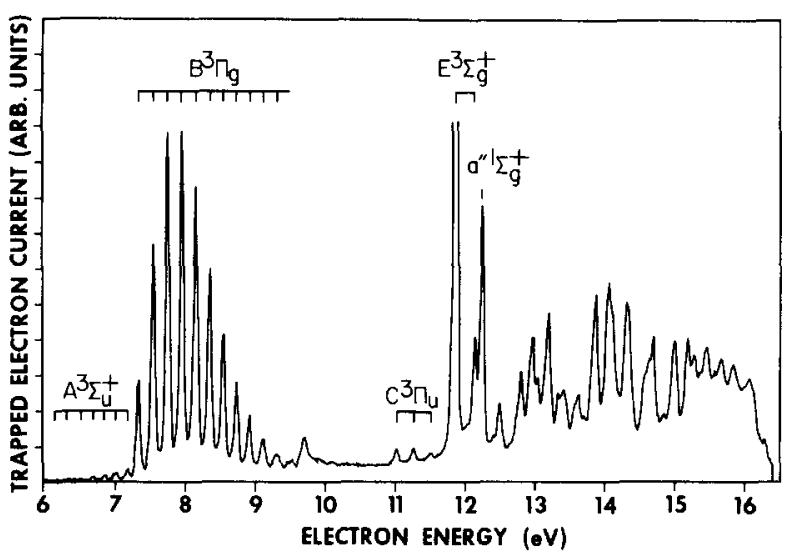

FIG. 4. Trapped electron current as a function of electron impact energy in nitrogen. The well depth is $\leqslant 15 \mathrm{mV}$.

in which one measures the trapped electron current generated by electron collisions over the full length of the collision chamber, the effective electron energy resolution is often substantially poorer than that indicated by the distribution emerging directly from the monochromator. This "internal" broadening ${ }^{21}$ is generally attributed to the fringe fields created near the end electrodes of the collision chamber and to nonuniformities in the surface potential within the chamber.

To minimize end effects, the collision chamber is disigned to collect trapped electrons over only one-third of its total length. This is accomplished by using only the middle pair of inner collision chamber electrodes as collectors and the outer pairs as guards whose potentials may be adjusted for the required mode of operation. A similar arrangement using a parallel-plate geometry was employed by Hall et al..$^{22}$

The use of a molecular beam rather than a static gas cell is unconventional in the trapped electron method. Nevertheless, a beam geometry offers several advantages since the volume in which collisions take place is smaller and thus the exposure to potential irregularities is reduced. In our apparatus, the molecular beam from the discharge passed primarily through the center pair of electrodes and the major portion of the scattering takes place in this relatively field-free region. For measurements at deep trap depths, a molecular beam geometry would eliminate the energy broadening which occurs in static cells due to collisions near the entrance plate where the electrons are being accelerated to their final energy.

To illustrate the performance of the apparatus in the trapped electron mode under optimum conditions, a spectrum of $\mathrm{N}_{2}$ in its ground state, over the energy range from 6 to $16 \mathrm{eV}$, is presented in Fig. 4. The spectrum was taken with a small well depth $\sim 15 \mathrm{mV}$ to indicate the best obtainable resolution of the trapped electron peaks. The results, in this regard, are somewhat improved over the best previously published data using magnetically collimated electron monochromators. ${ }^{22}$

\section{B. Excitation of the $B^{3} \Pi_{g}$ state near threshold}

Our trapped electron scheme for detection of $\mathrm{N}_{2}^{\mathrm{v}}$ is based on certain characteristics of the excitation of the $B^{3} \Pi_{g}$ 
state which we now discuss in more detail. Hall et al, ${ }^{22}$ in their trapped electron study of $\mathrm{N}_{2}$, were apparently the first to point out that the relative intensities of the peaks associated with the excitation of the $B^{3} I_{g}$ state near threshold were in good agreement with the calculated Franck-Condon factors for this transition.

Given that the transitions from $v=0$ to all $v^{\prime}$ of the $B$ ${ }^{3} I_{g}$ state are described accurately by the known FranckCondon factors, it is reasonable to assume that the transitions from higher vibrational levels of the ground state are similarly governed. A trapped electron spectrum taken in vibrationally excited $\mathrm{N}_{2}$ will thus contain progressions of peaks whose energies and peak envelopes are characterized by the transitions from each of the populated vibrational levels. Since the Franck-Condon factors are all known, the total trapped electron spectrum may be deconvoluted yielding the relative populations.

To examine carefully the extent to which the transitions from $v=0$ do in fact obey the Franck-Condon factors, and to provide a routine for deconvoluting the spectra taken in $\mathrm{N}_{2}^{V}$, a synthetic trapped electron spectrum was generated for detailed comparison with the data. The trapped electron peak heights cannot be compared directly to the FranckCondon factors since the peaks are not fully resolved. A simple means to determine the apparatus function is to extract the profile of a single trapped electron peak at a small well depth. In $\mathrm{N}_{2}$ this is very conveniently carried out using the threshold excitation to the $E^{3} \Sigma_{g}^{+}$state which exhibits essentially one well-isolated peak whose magnitude is a factor of 6 or more larger than neighboring features at small trapdepth. The digitized profile was used in a computer program which generated the total trapped electron spectra of the $A^{3} \Sigma_{u}{ }^{+}$ and $B^{3} I_{g}$ states, the principal components of the $\mathrm{N}_{2}$ spectrum over the energy range from 6 to $10 \mathrm{eV}$. The height of each individual vibrational peak of the given electron state was normalized to the appropriate Franck-Condon factor ${ }^{23}$ and the peaks spaced by the proper vibrational energy and anharmonicity. The peaks were then summed over energy to obtain a synthetic spectrum for each state separately. These

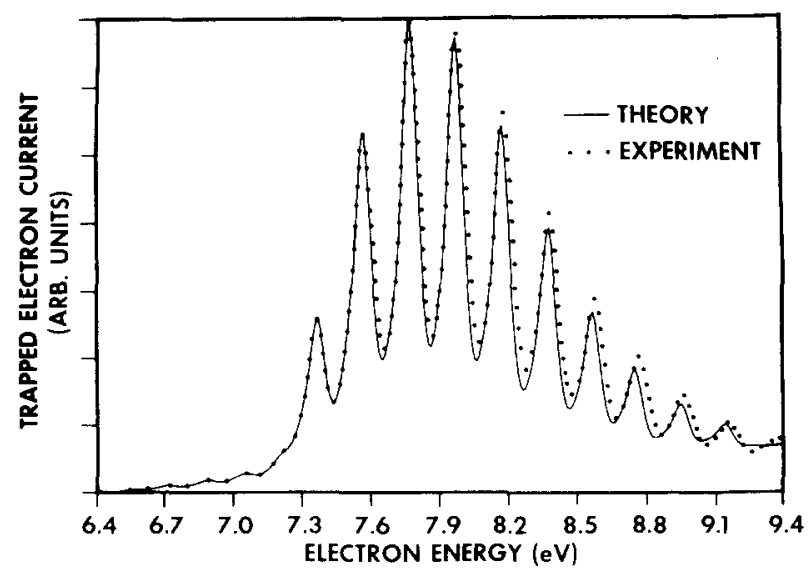

FIG. 5. Trapped electron spectrum of $\mathrm{N}_{2}(v=0)$ in the region of the $A^{3} \Sigma_{u}{ }^{+}$ and ${ }^{3} I_{g}$ states. The solid line is a computer generated spectrum described in the text. The noise in the experimental spectrum is less than the size of the dots. in turn, were then summed with the relative contributions of the $A^{3} \Sigma_{u}^{+}$and $B^{3} I_{\mathrm{g}}$ states adjusted to obtain the best match with the experimental spectrum. The best agreement was obtained when the $A^{3} \Sigma_{u}^{+}$contribution was $3.5 \%$ of that due to $B^{3} I_{g}$, reflecting the difference in the electronic portion of the excitation functions at threshold.

The computer generated trapped electron spectrum for $\mathrm{N}_{2}$ in its ground vibrational level, marked "theory," is compared with the experimental data ${ }^{24}$ in Fig. 5.. The agreement is excellent for the $A^{3} \Sigma_{u}^{+}$state and the lowest four vibrational levels of the $B^{3} \Pi_{g}$ state. At energies above $8 \mathrm{eV}$, the experimental points lie slightly above the theoretical curve. This is certainly due in part to the omission from the program of the $W^{3} \Delta_{u}$ state which has a maximum in its FranckCondon envelope near $8.5 \mathrm{eV} .{ }^{25}$ For the detection of $\mathrm{N}_{2}^{\mathrm{V}}$, it was not necessary to fit this portion of the spectrum and no attempt to include this or higher states was made. Further comments on the electron excitation of the $B^{3} I I_{g}$ state near threshold and the Franck-Condon factors are given in Appendix A.

Finally, we note that the data in Fig. 5 were taken with greater trap depth and hence lower peak resolution than shown in Fig. 4. For the detection of $\mathrm{N}_{2}^{\mathrm{V}}$, it was necessary to switch the discharge on and off, and shifts in contact potential precluded the use of such small trap depths.

\section{Detection of $N_{2}^{\frac{y}{2}}$}

A trapped electron spectrum in vibrationally excited $\mathrm{N}_{2}$ exhibits additional peaks analogous to the "hot bands" of optical spectroscopy. The peaks representing excitation of the $B^{3} \Pi_{\mathrm{g}}$ state from the vibrationally excited levels of the ground electronic state will be shifted below those produced from $v=0$ by the appropriate number of vibrational quanta. Furthermore, the Franck-Condon envelopes connecting each of the vibrational levels to the vibrational levels of the $B^{3} I I_{g}$ state have their own unique profiles. In addition to the presence of the new peaks at lower energy, the trapped electron spectrum of $\mathrm{N}_{2}^{\mathrm{v}}$ will therefore have a different overall envelope. We exploit these alterations in the trapped electron spectrum to detect $\mathrm{N}_{2}^{v}$.

The fitting procedure for $\mathrm{N}_{2}^{v}$ is similar to that for the ground state except that we must include the vibrational level populations as adjustable parameters. With the experimental conditions employed in the work to be described elsewhere, ${ }^{12}$ namely, discharge pressures near 6 Torr and transit times in excess of $5 \mathrm{msec}$ between the microwave cavity and the sampling slit, excellent fits to the trapped electron spectra were found using a Boltzmann distribution of vibrational levels at a single characteristic vibrational temperature. This is consistent with work of Black et al, ${ }^{8}$ who indicated that 0.5 Torr of $\mathrm{N}_{2}$ and a flow time of $20 \mathrm{msec}$ was more than sufficient to alter the initial distribution of vibrational levels produced in the discharge to one described by a single temperature. Under these conditions fitting the data is considerably simplified.

Figure 6 displays the experimental and theoretical trapped electron spectra for $\mathrm{N}_{2}$ at a pressure of 6 Torr subjected to a $100-\mathrm{W}$ microwave discharge. The excited gas is 


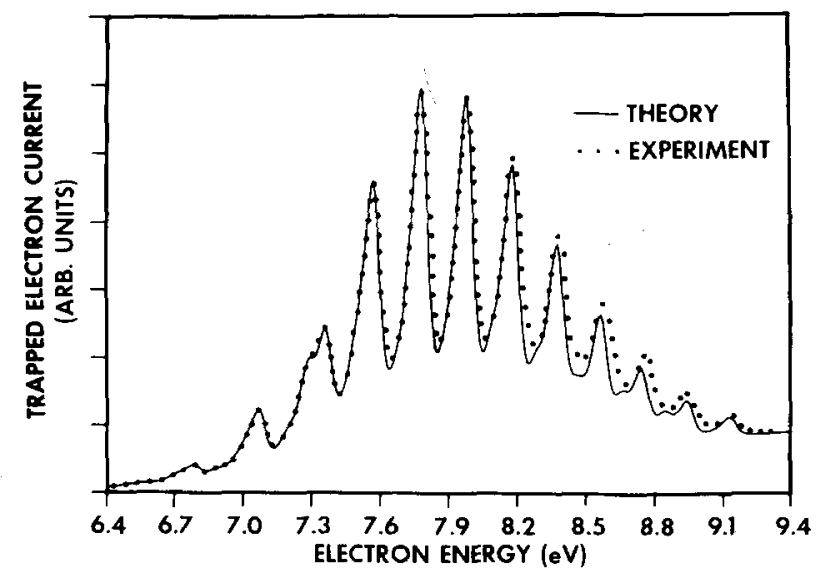

FIG. 6. Trapped electron spectrum of vibrationally excited $\mathrm{N}_{2}$ produced by a microwave discharge. The vertical scale is the same as in Fig. 5. The theoretical curve is in best agreement with the experiment for a Boltzmann distribution of vibrational levels characterized by a temperature of $1930 \mathrm{~K}$.

sampled after flowing for $8 \mathrm{msec}$ downstream from the discharge. Several new transitions into the $B{ }^{3} I I_{\mathrm{g}}$ state are apparent. The vertical scale in Fig. 6 is the same as that of Fig. 5 , and a substantial overall decrease in amplitude of the envelope is evident.

Although the experimental data were taken as a continuous function of energy, they are displayed in Figs. 5 and 6 as discrete points to separate them more clearly from the theoretical curve. The size of the dots is slightly greater than the peak to peak noise in the experimental data. In Fig. 6 the best agreement occurred for a vibrational temperature of $1930 \pm 50^{\circ} \mathrm{K}$. The fractional populations for $v=0,1,2$, and 3 are $0.824,0.145,0.026$, and 0.004 respectively, at this temperature.

The trapped electron method appears to be an accurate way of measuring the relative populations of the lower vibrational levels of $\mathrm{N}_{2}$, however, care has to be taken in obtaining spectra free from undesired instrumental effects. Because the trapping well is small, contact potential shifts caused by the discharged gas can alter the signal. In our experiment the contact potential shift manifests itself as a slow drift in the trapped electron current after the discharge has first been turned on. The drift however diminishes with time and disappears in about $15 \mathrm{~min}$. Upon turning the discharge off, the potentials do not revert to their original values for a long time, and no further difficulties are encountered in acquiring reproducible data with the discharge either on or off.

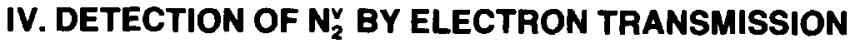

\section{A. The electron transmission method}

The electron transmission method ${ }^{14}$ provides a means to derive a signal related to the total electron scattering cross section as a function of electron energy. In $\mathrm{N}_{2}$ the total cross section $^{26}$ contains very pronounced resonant structure due to the formation of a temporary negative ion $\mathrm{N}_{2}^{-}\left(\mathrm{X}^{2} I I_{g}\right)$ at energies near $2 \mathrm{eV}$. The electron transmission method is extremely sensitive to the energies and shapes of these narrow features. In vibrationally excited $\mathrm{N}_{2}$, the locations and magnitudes of the resonant sturctures are altered from those ob- served from the ground vibrational level and we exploit these differences to detect the excited species.

In the electron transmission mode, a beam of electrons is directed through the scattering gas at a density sufficient to cause an appreciable attenuation of the electron beam current. The scattered electrons are rejected by a retarding voltage which discriminates against their reduced axial component of velocity. The unscattered current "transmitted" to the electron beam collector is therefore given by

$$
I(E)=I_{0} \exp [-\mathrm{NQ}(\mathrm{E}) \mathrm{L}],
$$

where $I_{0}$ is the incident electron beam current, $N$ is the gas density, $L$ is the scattering path length, and $Q(E)$ is the total scattering cross section. Sharp structure in the energy dependence of the cross section will therefore be reflected in the variation of the transmitted current.

A substantial improvement in the sensitivity for detection of sharp structures in the cross section was introduced by Sanche and Schulz ${ }^{14}$ who measured the derivative of the transmitted current with respect to energy. The experimental technique for carrying out these measurements has been discussed in the literature. ${ }^{14} \mathrm{~A}$ bibliography of the papers imploying transmission spectroscopy for the study of temporary negative ions is available from one of the authors (PDB).

\section{B. The electron transmission spectrum of $\mathrm{N}_{2}$ near $2 \mathrm{eV}$}

To put transmission measurements on an absolute basis, the total scattering cross sections of $\mathrm{N}_{2}$ in its ground and excited vibrational levels are required in the energy range over which the temporary negative ion is formed. Experimental studies of the resonance and its decay properties have been reviewed by Schulz. ${ }^{27}$ Unfortunately, total cross section measurements from the vibrationally excited states are not available. On the theoretical side, a quantitatively correct description of the fine structure in the cross section has proved to be a considerable challenge. Theoretical studies in $\mathrm{N}_{2}$ have been discussed recently by Dubé and Herzenberg. ${ }^{15}$ The most accurate reproduction of the fine structure in the total scattering cross section from $v=0$ of $\mathrm{N}_{2}$, as indicated by direct comparison with experimental data, is obtained with the "boomerang" model of Herzenberg and his coworkers. ${ }^{28}$ As recently updated by Dubé and Herzenberg, ${ }^{15}$ this semiempirical model is parameterized by fitting to recent electron impact measurements of vibrational excitation by Wong et al. ${ }^{29}$ Certain aspects of the resonant structure in the cross section, its appearance in the transmission spectrum, and the lifetime of the temporary negative ion are discussed in more detail in Appendix B.

The calculations of Dubé and Herzenberg ${ }^{15}$ yield only the resonant portion of the scattering cross section, that is, the contribution proceeding through the intermediate formation of $\mathrm{N}_{2}^{-}\left({ }^{2} I I_{g}\right)$. To simulate the total cross section, we have added a constant nonresonant contribution of $11 \AA^{2}$. This value was obtained by averaging through the off-resonant portion of the total electron scattering cross section measured by Golden. ${ }^{26}$ Although the assumption that the nonresonant contribution is energy independent is not strictly correct, it simplifies the computation and, as discussed 


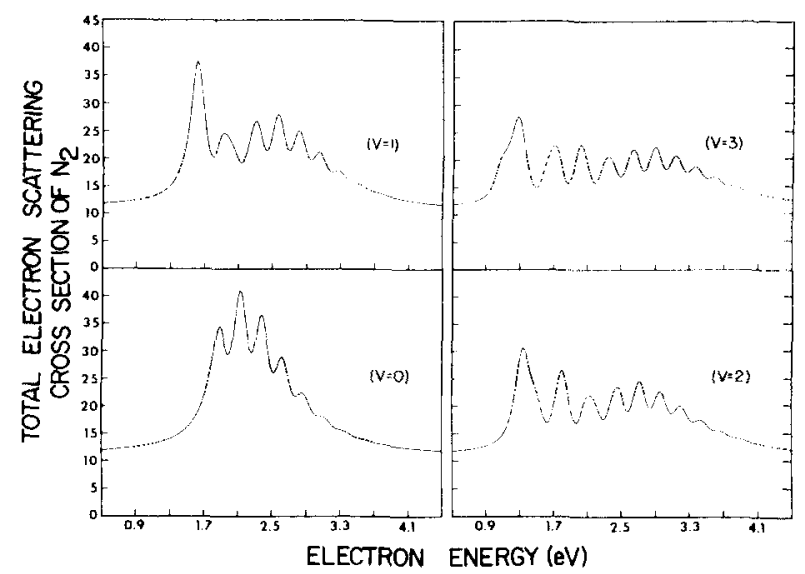

FIG. 7. Total scattering cross sections in $\AA^{2}$ for $\mathrm{N}_{2}$ in $v=0,1,2$, and 3 as a function of energy as computed by Dube and Herzenberg using the boomerang model. A constant nonresonant cross section of $11 \AA^{2}$ has been added to the resonant contribution.

below, does not greatly affect the comparison between theory and experiment for the transmission data taken in the derivative mode. In fact, our assumed nonresonant cross section is in good agreement with that calculated by Chandra and $\mathrm{Temkin}^{30}$ who found it to be only weakly varying over the range of energies in which $\mathrm{N}_{2}^{-}$is formed. The total scattering cross section from $v=0$ in the vicinity of the $2-\mathrm{eV}$ resonance, derived as described above, is displayed in the lowest panel of Fig. 7.

We now examine the extent to which the experimental electron transmission spectrum of ground-state $\mathrm{N}_{2}$ in the resonance region agrees with the theoretical cross section. To generate theoretical values for the derivative of the transmitted current, we must specify the particular values used for the density of the scattering gas, and the scattering pathlength. The product $N L$ is determined experimentally by measuring the ratio of the transmitted current with and without the scattering gas at energy $E_{0}$ and substituting into

$$
N L=\left[1 / Q\left(E_{0}\right)\right] \ln \left[I_{0} / I\left(E_{0}\right)\right] .
$$

With $N L$ determined, we then compute $d I / d E$ and normalize the result to the experimental transmission spectrum.

Figure 8 shows the agreement between theory and experiment for ground-state $\mathrm{N}_{2}$. The computer generated spec-

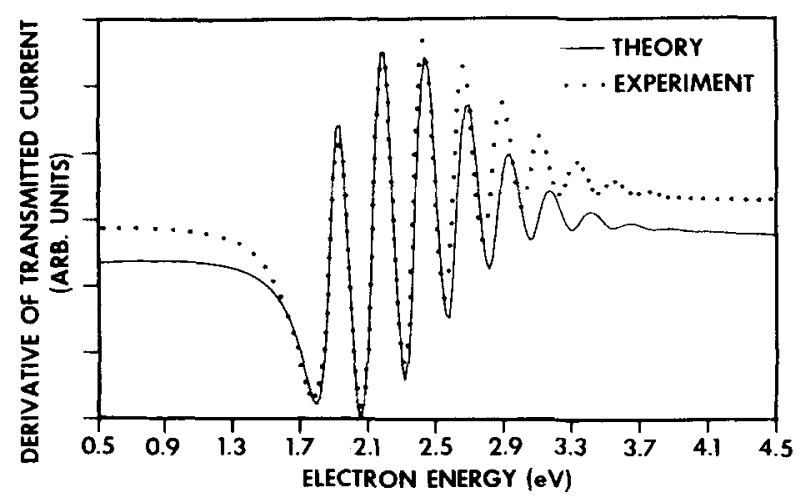

FIG. 8. Derivative of electron current transmitted through $\mathrm{N}_{2}$ as a function of energy. The theoretical curve is computed using the total scattering cross section for $v=0$ shown in Fig. 7. trum is shown as a continuous line, while the experimental spectrum is dotted. The noise in the experimental data is less than the size of the dots. The derivative transmission method is very sensitive to the widths and slopes of the sharp structure in the cross sections. Thus the important criterion in judging the quality of the comparison is the accuracy of the reproduction of the relative distances between peaks and dips in the derivative spectrum. Because of the assumption that the nonresonant contribution to the total electron scattering cross section is a constant, the theoretically generated spectrum does not match the background observed in the experiment away from the resonance. To facilitate the visual comparison between theory and experiment, a sloping background has been added to the computed spectrum.

Figure 8 indicates that the agreement between theory and the experiment with respect to the sharp structure is rather good. The relative peak to dip distances reproduce the experimental structure quite accurately. The most pronounced difference between the two spectra occurs at energies above $2.5 \mathrm{eV}$, where the "phases" of the oscillations do not match. These shifts are due to the neglect of anharmonicity in the functions used to model the potential curves of $\mathrm{N}_{2}$ and $\mathrm{N}_{2}^{-}$. The extreme sensitivity of the results to the potential curves is discussed by Dubé and Herzenberg. ${ }^{15}$ Although particular attention was paid to the accuracy at low energies by these authors, there were only a limited number of empirically determined parameters used to model the curve.

\section{Detection of $\mathrm{N}_{2}^{\vee}$}

In this section we extend the discussion to $\mathrm{N}_{2}^{v}$ and demonstrate that the transmission spectra of vibrationally excited nitrogen can be used to extract the vibrational population.

For each of the vibrational levels present, we require the total scattering cross section in the resonance region. The theoretical cross sections of Dubé and Herzenberg ${ }^{15}$ for $\mathrm{N}_{2}$ in $v=1,2$, and 3 are shown in the other panels of Fig. 7. In each case the resonant contribution has been added to a constant $11 \AA^{2}$ nonresonant portion assumed to be the same for each level. The fitting procedure is similar to that using the trapped electron method. The total scattering cross sections, weighed by the relative populations associated with a given vibrational temperature, are summed over energy. The computer program then calculates the derivative of the transmitted current as described previously.

Figure 9 illustrates the fit to a transmission spectrum taken under the following conditions: microwave discharge power of $180 \mathrm{~W}$, pressure in the discharge region of 6 Torr, transit time from cavity to the electron scattering region of 8 msec. The spectrum was taken at the same total pressure of scattering gas as that of $\mathbf{N}_{2}(v=0)$ as shown in Fig. 8. The vertical scales in these two figures are the same, that is, the theoretical curve is normalized to experiment only in Fig. 8. The overall envelope of the peaks is reduced from that found in the undischarged gas and additional pronounced structure is seen at energies below $1.7 \mathrm{eV}$. The new peaks arise from the formation of the temporary negative ion from the 


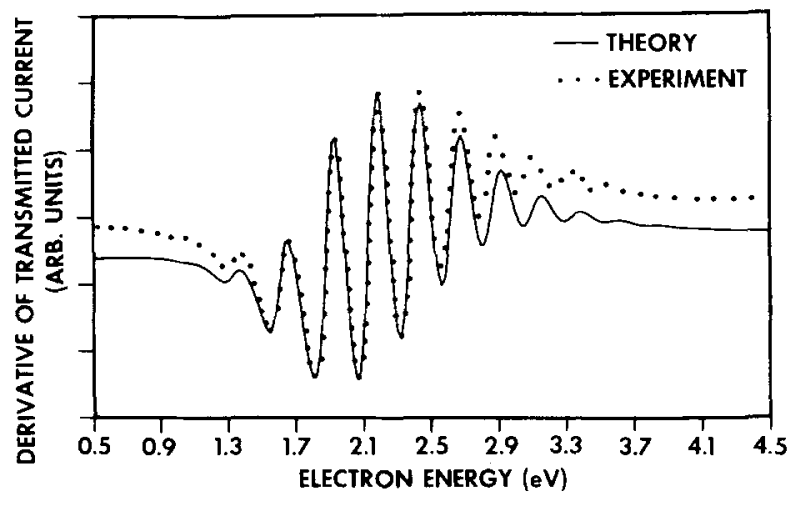

FIG. 9. Derivative of transmitted current in vibrationally excited $\mathrm{N}_{2}$ produced by a microwave discharge. The vertical scale is the same as that of Fig. 8. The theoretical curve is in best agreement with experiment for a Boltzmann distribution of vibrational levels characterized by a temperature of $2450 \mathrm{~K}$.

excited vibrational levels of the neutral ground state and are more transparently seen in the theoretical cross sections of Fig. 7. Again, the quality of the agreement between theory and experiment for the discharged gas, as reflected in the relative peak to dip distances, is quite good. Using the vibrational temperature as a parameter, the best fit between theory and experiment was found for a temperature of $2450 \pm 600^{\circ} \mathrm{K}$

\section{DISCUSSION}

The trapped electron and transmission methods can both be used to obtain quantitative estimates of the populations of the lowest few vibrational levels of excited $\mathrm{N}_{2}$. The trapped electron method, by virtue of the simplicity of the interpretation, is considered here to be more reliable. The errors in the fitting of the transmission spectrum depend primarily on the accuracy of the theoretical total electron scattering cross sections from $\mathrm{N}_{2}$ in $v=1,2$, and 3 , and this at present is not known.

As a partial check of the transmission method, an experiment was carried out in which the vibrational temperature was measured by both methods under identical discharge conditions. The measurements were made for eight different microwave power levels ranging from 20 to $180 \mathrm{~W}$. The data were taken at a fixed discharge pressure of 6 Torr and a transit time from discharge to scattering chamber of 8 msec.

The results are shown in Fig. 10 which displays the derived vibrational temperature as a function of microwave power. The error bars in the trapped electron fit were obtained from the assumption that the percentage of $v=1$ content can be fitted to $0.5 \%$ of the total nitrogen density. The error in the transmission method, approximately $20 \%$, was derived from the discrepancies between theory and experiment in the fit for $v=0$. Within these error limits, the two techniques are quite consistent.

Although the trapped electron and transmission methods are each useful for detection of $\mathrm{N}_{2}^{\vee}$, the trapped electron method is intrinsically more accurate because fitting of the spectrum utilizes Franck-Condon factors which are well es-

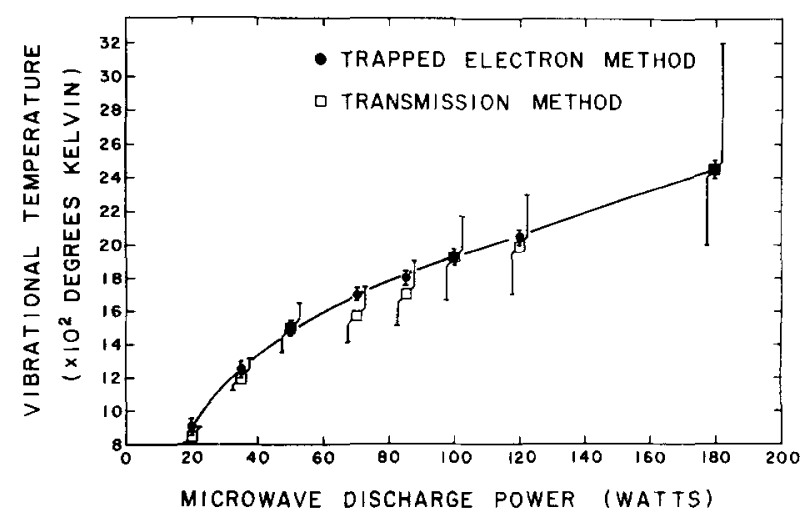

FIG. 10. Vibrational temperature of excited nitrogen produced by a microwave discharge as a function of discharge power. The temperatures are measured independently using the trapped electron and transmission methods.

tablished. In addition to the appearance of new peaks, the overall change in the peaks corresponding to excitation of the higher vibrational levels of the $B$ state provides a consistency check. On the other hand, the trapped electron method is more difficult experimentally because of its smaller signals and sensitivity to changing surface potentials and spurious background currents.

The transmission method is less accurate because of the uncertainty in the total scattering cross sections and because of the difficulties with making point to point comparisons between theory and experiment. Experimentally, the technique is more rugged and less prone to drifts and spurious signals, and can be used under more adverse conditions. Using transmission, for example, we were able to observe vibrationally excited $\mathrm{N}_{2}$ produced in a dc discharge placed only 2 $\mathrm{cm}$ from the electron beam. Under these circumstances the trapped electron spectra were overwhelmed by large background currents and could not produce meaningful results. Because the resonant peaks in the scattering cross section are so pronounced and relatively broadly spaced, high-energy resolution is not required in the electron monochromator. Indeed, observation of $\mathrm{N}_{2}^{v}$ could be made in the transmission mode using the unmonochromated beam of electrons emerging directly from the filament. ${ }^{31}$

Note added in proof. Related work has recently been published by A. Huetz, F. Gresteau, R. I. Hall, and J. Mazeau, J. Chem. Phys. 72, 5297 (1980).

\section{ACKNOWLEDGMENTS}

This work was supported by NSF Grant ENG 7102474 and ENG 77-14649.

\section{APPENDIX A}

In this appendix we point out that the agreement of the $B^{3} \Pi_{g}$ trapped electron peaks with the calculated FranckCondon factors is not well understood. At energies at which excitation to a given electronic state is "direct," that is, does not proceed through an intermediate temporary negative ion, such behavior would be expected. Near threshold, however, the excitation of electronic states may be influenced by core-excited, or Feshbach, resonances lying just below their 
"parent" state, ${ }^{27}$ and in such a case the trapped electron spectrum would not be expected to follow the spectroscopic Franck-Condon factors. On the other hand, the role of resonances in the threshold excitation of valence states, which do not support Feshbach resonances according to calculations of Weiss and Krauss, ${ }^{32}$ is considerably less certain. The differential excitation functions of the $A^{3} \Sigma_{u}$ and $B^{3} I_{8}$ valence states of $\mathrm{N}_{2}$ have been examined by Mazeau et al. ${ }^{3.3}$ who find them to be dominated by several broad overlapping resonant peaks. These authors attribute these features to core-excited shape resonances, that is, temporary negativeion states which lie above the neutral excited state and which decay primarily into the parent state. Near threshold Mazeau et al. ${ }^{33}$ report that the $B{ }^{3} \mathrm{II}_{z}$ excitation function rises rapidly reaching a small peak very near threshold, and then continuing upward to larger and broader maxima. In addition, the threshold excitation is characterized by an isotropic angular dependence. If the threshold behavior is caused by an s-wave core-excited shape resonance as suggested by $\mathrm{Ma}$ zeau et al. ${ }^{3,3}$ the agreement between the trapped electron data and the Franck-Condon factors is indeed puzzling. One possible explanation could be that the potential curve for the negative-ion state is virtually identical to and superposed on that of the neutral molecule in its excited state. Further examination of this problem is in order, particularly a study of the extent to which the Franck-Condon factors are obeyed as the energy above threshold is increased.

\section{APPENDIX $B$}

The electron transmission method has been extensively used to locate temporary negative ions in atoms and small molecules. ${ }^{34}$ In molecules, the presence of vibrational structure in the spectrum is particularly useful. If the structure is sufficiently sharp, the vibrational modes of the negative ion and the adiabatic and vertical electron affinities of the molecule may be determined in many cases. The visibility of the vibrational structure is strongly tied to the lifetime of the anion. Dube and Herzenberg, ${ }^{15}$ using the boomerang model, derive a value of $\Gamma_{0}=0.54 \mathrm{eV}$ for the spread in energy associated with the finite lifetime of $N_{2}-$ at its equilibrium internuclear separation. This decay width is approximately twice the anionic vibrational level spacing in the absence of autoionization. The boomerang model is therefore appropriate for $\mathrm{N}_{2}$ since it allows the $\mathrm{N}_{2}^{-}$wave packet to describe only one outgoing and one returning portion of an oscillation before the negative-ion autoionizes. The features in the cross section, and hence in the transmission spectrum, are therefore not true vibrational structures of the anion. Rather, they arise, in a time dependent picture, from interference between the outgoing nuclear wave packet and that portion of the wave packet which survives to the turning point and reflects. At best the structure may be regarded as "quasivibrational" in nature. ${ }^{2 \mathrm{~s}}$

From our experience in dealing with a broad range of molecules, particularly unsaturated hydrocarbons, ${ }^{34}$ it appears that the majority possess quasivibrational structure in their transmission spectra which is less pronounced than that of $\mathrm{N}_{2}$. The boomerang approach, if suitably generalized

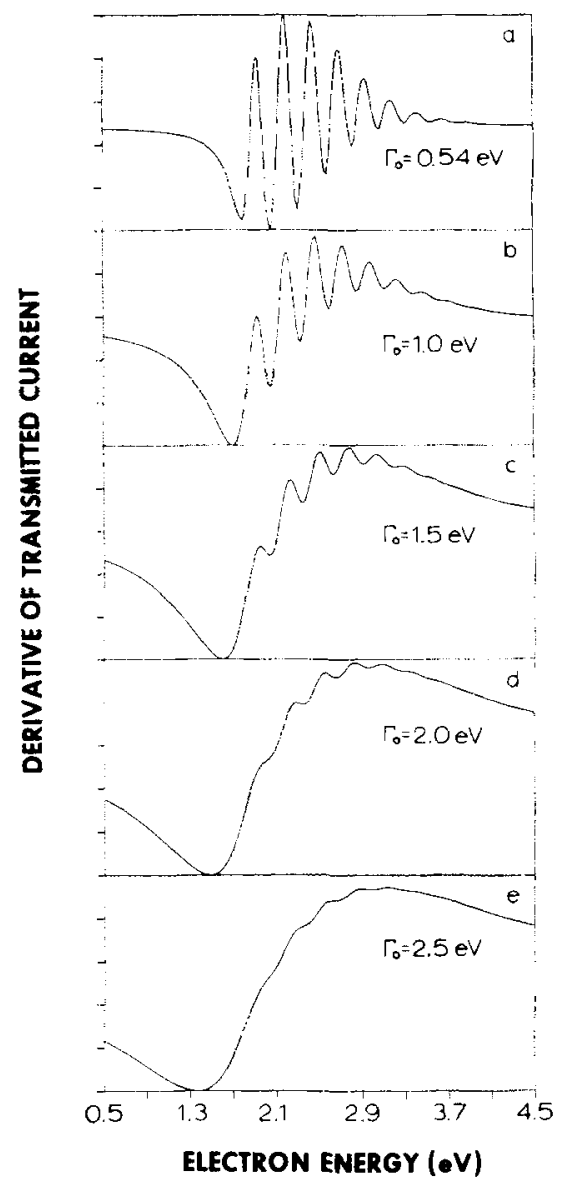

FIG. 11. Derivative of transmitted current in $\mathrm{N}_{2}$ as a function of energy computed with the boomerang model. (a) All parameters as derived by Dubé and Herzenberg, $\Gamma_{0}=0.54 \mathrm{eV}$, (b) $\Gamma_{0}=1.0 \mathrm{eV}$, (c) $\Gamma_{0}=1.5 \mathrm{eV}$, (d) $\Gamma_{0}=2.0 \mathrm{eV}$, and $(\mathrm{e}) \Gamma_{\mathrm{B}}=2.5 \mathrm{eV}$.

to polyatomic surfaces, may therefore provide a generally useful and appropriate basis for describing shape resonances in these molecules.

For purposes of comparison, it is useful to examine the range of anion lifetimes over which quasivibrational structure perseveres in the electron scattering cross section. As a by-product of the calculations generating the resonance cross sections in $\mathrm{N}_{2}$, it was possible to simulate anions of shorter lifetime, leaving all other parameters except $\Gamma_{0}$ fixed as they were derived by Dubé and Herzenberg. ${ }^{15}$ In Fig. $11(a)$, we show the computer generated transmission spectrum of $\mathrm{N}_{2}$, that is, the derivative of electron current transmitted through $\mathrm{N}_{2}$ as a function of energy, with all parameters as derived by Dubé and Herzenberg. ${ }^{15}$ Again, only the resonant portion of the total scattering cross section is included. The successive curves shown in Figs. $11(\mathrm{~b})$ and $11(\mathrm{e})$ display the spectra with $\Gamma_{0}$ increased to $1.0,1.5,2.0$, and 2.5 $\mathrm{eV}$, respectively. Surprisingly, faint structure in the cross section is still visible even for $\Gamma_{0}=2.5 \mathrm{eV}$. The nitrogen negative ion may be somewhat anomalous in this regard because of its steep potential curve in the Franck-Condon region of the neutral molecule.

The visibility of the quasivibrational structure in certain of these curves is similar to that measured in other gases. In particular the transmission spectrum ${ }^{34}$ of $\mathrm{CO}$ is quite close to that of Fig. 14(d), and ethylene ${ }^{35}$ to that in Fig. 11(e). 
It would appear that the boomerang model extended to polyatomic surfaces will provide a useful means to extract lifetime information in cases in which faint structure due to nuclear motion occurs.

'F. Kaufman and J. R. Kelso, J. Chem. Phys. 28, 510 (1958); J. E. Morgan and H. I. Schiff, Can. J. Chem. 41, 903 (1963).

${ }^{2} \mathrm{~K}$. Dressler, J. Chem. Phys. 36, 281 (1959).

${ }^{3}$ S. N. Foner and R. L. Hudson, J. Chem. Phys. 45, 40 (1966).

${ }^{4}$ G. R. Cook and R. J. McNeal, J. Chem. Phys. 56, 1388 (1972).

${ }^{5}$ M. H. Bruce, A. T. Stair, Jr., and J. P. Kennealy, J. Chim. Phys. 64, 36 (1967); K. P. Horn and P. E. Oettinger, J. Chem. Phys. 54, 3040 (1971).

'A. L. Schmeltekopf, E. E. Ferguson, and F. C. Fehsenfeld, J. Chem. Phys. 48, 2966 (1968)

${ }^{7}$ E. P. Muntz and D. J. Marsden, Rarified Gas Dynamics Symposium II (Academic, New York, 1963), pp. 495-526.

${ }^{8}$ L. Y. Nelson, A. W. Saunders, Jr., A. B. Harvey, and G. O. Neely, J. Chem. Phys. 55, 5127 (1971); G. Black, R. L. Sharpless, and T. G. Slanger,

J. Chem. Phys. 58, 4792 (1973).

${ }^{9}$ J. W. Nibler, J. R. McDonald, and A. B. Harvey, Opt. Commun. 18, 371 (1976)

${ }^{10}$ J. Dyke, N. Jonathan, A. Morris, and T. Sears, J. C. S. Faraday Transactions II 72, 597 (1976).

I'M. D. White and K. J. Ross, J. Phys. B 9, 2147 (1976).

'2J. A. Michejda and P. D. Burrow (unpublished).

${ }^{13}$ G. J. Schulz, Phys. Rev. 112, 150 (1958).

${ }^{14} \mathrm{~L}$. Sanche and G. J. Schulz, Phys. Rev. A 5, $1672(1972)$

${ }^{15}$ L. J. Dubé and A. Herzenberg, Phys. Rev. A 20, 194 (1979).

${ }^{16}$ P. D. Burrow, J. Chem. Phys. 59, 4922 (1973); L. Sanche and P. D. Burrow, Phys. Rev. Lett. 29, 1639 (1972).

${ }^{17}$ A. Wright and C. Winkler, Active Nitrogen (Academic, New York, 1968).
${ }^{18} \mathrm{G}$. Black, H. Wise, S. Schechter, and R. L. Sharpless, J. Chem. Phys. 60 , 3526 (1974).

${ }^{19}$ A. Stamatovic and G. J. Schulz, Rev. Sci, Instrum. 41, 423 (1970).

${ }^{20}$ P. D. Burrow and G. J. Schulz, Phys. Rev. 187, 97 (1969); D. Spence, J. L. Mauer, and G. J. Schulz, J. Chem. Phys. 57, 5516 (1972).

${ }^{21}$ G. J. Schulz, Phys. Rev. 136, 650 (1964).

${ }^{22}$ R. I. Hall, J. Mazeau, J. Reinhardt, and C. Schermann, J. Phys. B 3, 991 (1970).

${ }^{23}$ W. Benesch, J. T. Vanderslice, S. G. Tilford, and P. G. Wilkinson, Astrophys. J. 142, 1227 (1965).

${ }^{24}$ The experimental spectrum is superposed on a small, gently rising background due to elastically scattered electrons which reach the collector. To allow a more direct visual comparison of theory and experiment, a linearly increasing function is added to the computer generated spectra to simulate this background. A straight line was found to be an adequate representation of the background in helium, where any current collected at energies below the first threshold for excitation must be due to elastically scattered electrons.

${ }^{25}$ K. A. Saum and W. M. Benesch, Phys. Rev. A 2, 1655 (1970); W. M. Benesch and K. A. Saum, J. Phys. B 4, 732 (1971).

${ }^{2 n}$ D. E. Golden, Phys. Rev. Lett. 17, 847 (1966)

${ }^{27}$ G. J. Schulz, Rev. Mod. Phys. 45, 378 (1973).

${ }^{28}$ A. Herzenberg, J. Phys. B 1, 548 (1968); D. T. Birtwistle and A. Herzenberg, J. Phys. B 4, 53 (1971).

${ }^{29}$ S. F. Wong, J. A. Michejda, and A. Stamatovic (unpublished).

${ }^{30} \mathrm{~N}$. Chandra and A. Temkin, Phys. Rev. A 13, 188 (1976).

"J. A. Michejda and P. D. Burrow, J. Appl. Phys. 47, 2780 (1976).

${ }^{32}$ A. W, Weiss and M. Krauss, J. Chem. Phys, 52, 4363 (1970).

${ }^{33}$ J. Mazeau, F. Gresteau, R. I. Hall, G. Joyez, and J. Reinhardt, J. Phys. B 6, $862(1973)$.

${ }^{34}$ Applications to larger hydrocarbon molecules are reviewed in K. D. Jordan and P. D. Burrow, Acc. Chem. Res. 11, 341 (1978).

${ }^{35}$ P. D. Burrow and K. D. Jordan, Chem. Phys. Lett. 36, 594 (1975). 